\title{
ON THE ADEQUACY OF SHELL MODELS FOR PREDICTING STRESSES AND STRAINS IN THICK-WALLED TUBES SUBJECTED TO DETONATION LOADING
}

\author{
Neal P. Bitter and Joseph E. Shepherd \\ Explosion Dynamics Laboratory \\ California Institute of Technology \\ Pasadena, California 91125 \\ Email: nbitter@caltech.edu
}

\begin{abstract}
This paper analyzes the adequacy of shell models for predicting stresses and strains in thick-walled tubes subjected to detonation loads. Of particular interest are the large axial strains which are produced at the inner and outer surfaces of the tube due to bending along the tube axis. First, comparisons between simple shell theory and a static finite element model are used to show that the axial strain varies proportionally with wall thickness and inversely with the square of the axial wavelength. For small wavelengths, this comparison demonstrates nonlinear behavior and a breakdown of the shell model. Second, a dynamic finite element model is used to evaluate the performance of transient shell equations. This comparison is used to quantify the error of the shell model with increasing wall thickness and show that shell models can be inaccurate near the load front where the axial curvature is high. Finally, the results of these analyses are used to show that the large axial strains which are sometimes observed in experiments cannot be attributed to throughwall bending and appear to be caused instead by non-ideal conditions present in the experiments.
\end{abstract}

\section{NOMENCLATURE}

a Mean tube radius

$h$ Wall thickness

E Elastic modulus

$G$ Shear modulus $E / 2(1+v)$

$L$ Tube length

$u$ Axial displacement

\author{
$v_{d}$ Dilatational Wave speed $\sqrt{E / \rho\left(1-v^{2}\right)}$ \\ $v_{s}$ Shear wave speed $\sqrt{\kappa G / \rho}$ \\ $v_{c o} \quad$ First critical wave speed \\ $w$ Radial displacement \\ $w_{b}$ Component of radial displacement due to axial bending \\ $w_{s}$ Component of radial displacement due to transverse shear \\ $z$ Radial distance from middle surface of tube \\ $\beta$ Shell thickness parameter $h / \sqrt{12} a$ \\ $\varepsilon_{\theta} \quad$ Hoop strain \\ $\varepsilon_{x} \quad$ Axial strain \\ $\kappa$ Shear correction factor, taken to be $5 / 6$ \\ $v$ Poisson's ratio \\ $\rho$ Density of tube \\ $\psi_{x}$ Angular deformation of tube
}

\section{INTRODUCTION}

Linear, elastic, thin-shell theories have formed the foundation for our current understanding of the dynamic response of cylindrical tubes and pipes. Although a large number of variations on the thin-shell equations have been proposed [1], most of them are based on a set of assumptions commonly called "Love's first approximation" [2]. These assumptions are the following:

1. The wall thickness $h$ is much smaller than any other dimension of the shell, including the radii of curvature

2. Non-linear terms in the strain-displacement relations are negligible

3. Transverse normal stress is negligibly small 
4. During deformation, normals to the undeformed middle surface remain straight and normal and their lengths are preserved (the Kirchhoff hypothesis)

Shell formulations based on these assumptions have been used very successfully to predict the behavior of thin tubes subjected to internal detonations and shock waves. For instance, Beltman et al. used a steady-state shell model to analyze peak strains in thin $(a / h=16)$ tubes subjected to internal shock waves and found reasonably good agreement with finite element models and experimental measurements except in the vicinity of the first critical velocity, where effects of material damping are important and the steady-state approximation is inadequate [3, 4]. Likewise Simkins measured hoop strains in a much thicker tube $(a / h=5.2)$ and found good agreement with the predictions of shell theory [5]. However, recent experiments [6] with thickwalled tubes $(a / h=7.2)$ have demonstrated axial strains that are 2-3 times larger than would be expected on the basis of thin shell models, even though hoop strains are well-predicted. This discrepancy calls for a thorough evaluation of the axial strains that develop in thick tubes subjected to shock or detonation type loads.

A possible explanation for the differences between experimental measurements and the predictions of shell models is that these models may no longer be adequate for tubes of large wall thickness. Thus one objective of this paper is to quantify how rapidly errors in shell models grow when the thin-wall approximation is violated. The thickness of a tube must be evaluated in comparison to other length scales, including the radii of curvature of the tube, and the tube can only be considered thin if the wall thickness is much smaller than all other dimensions. The most obvious scenario which breaks this constraint is that of a tube with wall thickness $h$ comparable to the undeformed radius of curvature $a$. However, for both thick and thin tubes subjected to dynamic loads, it is also possible for the axial radius of curvature to become comparable to the wall thickness, which is a second mechanism by which the thin-wall assumption might fail. Both of these cases are considered in this paper.

Various authors have evaluated the performance of thin-shell theories using comparisons with the three-dimensional equations of elasticity [7-10]. These comparisons are usually made by assuming displacements in the form of harmonic vibrations and comparing the dispersion curves from shell models with those from 3D elasticity. For instance, Herrmann and Mirsky proposed a shell model which included the effects of both shear deformation and rotary inertia and showed that the lowest branch of the dispersion curve for this model differed negligibly from that of 3D elasticity for all wavenumbers [10]. However, as pointed out by Greenspon [9], for high frequency vibrations the displacements may vary nonlinearly through the shell thickness and outer-surface stresses and strains can be in considerable error even when vibration frequencies are well-predicted.
Although the usual free-vibration analysis provides a simple framework in which solutions to the $3 \mathrm{D}$ equations of elasticity are tractable, it also involves a considerable departure from realistic loads, such as impulsive loads or shock waves, which are more commonly encountered in practice. The structural response of tubes subjected to shock and detonation loads often features a dynamic load amplification $[4,11,12]$ that leads to large stresses and strains near or behind the load front. The magnitude of these stresses and strains cannot be quantified using free-vibration analysis.

This paper seeks to evaluate the performance of shell equations for thick tubes in the specific context of shock or detonation type loads. Emphasis is placed on the prediction of stresses and strains at the inner and outer surfaces of the tube, which are often predicted less accurately than vibration frequencies or middlesurface deformation. In the first part of this paper, static finite element modeling is used to investigate the relationship between the axial curvature of the deformation and the resulting axial strains that are developed at the inner and outer surfaces of a tube. In the second part of this paper, the predictions of dynamic shell equations for shock wave type loads are evaluated through comparisons with finite element simulations.

It is worth noting that much of the discussion in this paper regarding thin shell models is also applicable to finite element simulations. Many types of shell elements are based on thin shell models and assume linear strain variation through the element thickness. As a result these models are subject to restrictions on wall thickness that are similar to those of thin shell theory. These restrictions can only be avoided by using several elements or integration points through the wall thickness in order to capture non-linear strain variation.

\section{STRAIN PREDICTIONS FROM SHELL MODELS}

A diagram of an axisymmetrically bending tube is shown in Fig. 1. The tube has a mean radius of $a$ and a wall thickness of $h$ and is deformed into a series of waves of wavelength $\lambda$ along the tube axis. The thin-shell approximation involves the construction of a local coordinate system along the middle surface of the tube (dashed line) such that $z=r-a$ is the radial distance from the middle surface and $x$ is the coordinate along the tube axis. Displacements in the radial and axial directions are denoted $w$ and $u$, respectively.

For axisymmetric deformation, the only non-zero components of strain at a point $(x, z)$ on the tube are given by:

$$
\begin{array}{ll}
\varepsilon_{z}=\frac{\partial w}{\partial z} & \varepsilon_{\theta}=\frac{w}{a+z} \\
\varepsilon_{x}=\frac{\partial u}{\partial x} & \varepsilon_{z x}=\frac{1}{2}\left(\frac{\partial u}{\partial z}+\frac{\partial w}{\partial x}\right)
\end{array}
$$




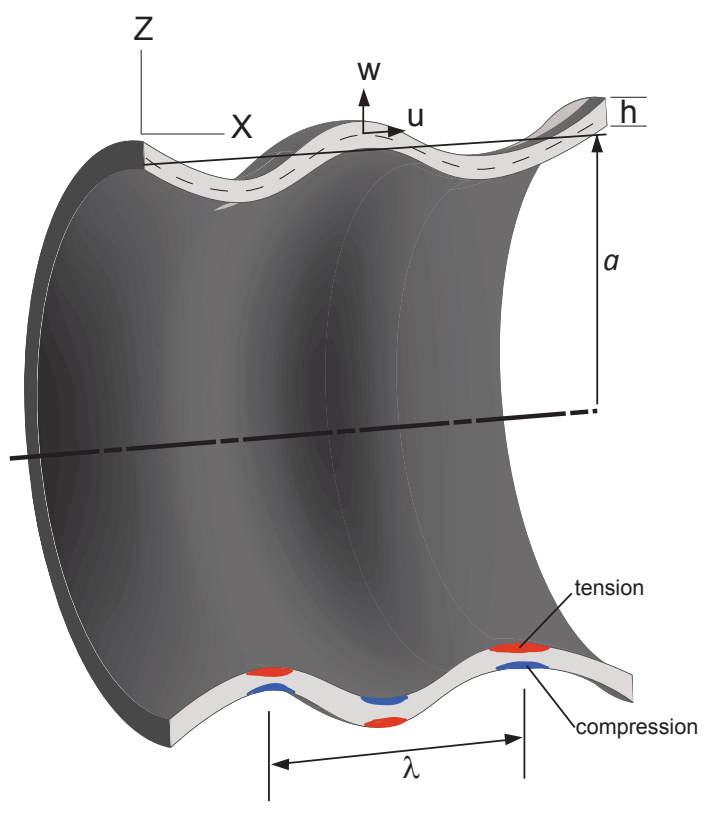

FIGURE 1. Axisymmetric deformation of a tube with a wavelength $\lambda$ of flexure. The coordinate system $(x, z)$ is a local construction with $z$ parallel to the radial direction and $x$ to the axial direction. The mean radius is $a$, and the distance $z$ is measured from the shell middle surface (dashed line).

In these expressions, shell approximations have not yet been introduced, so these strains are subject only to the constraint of small deformations, i.e., the products of displacement gradients are much smaller than the displacement gradients themselves.

Suppose that the displacements $w$ and $u$ are expressed using Taylor series expansions centered at the middle surface:

$$
\begin{gathered}
u(x, z)=u_{o}(x)+\left.\frac{\partial u}{\partial z}\right|_{z=0} z+O\left(z^{2}\right) \\
w(x, z)=w_{o}(x)+\left.\frac{\partial w}{\partial z}\right|_{z=0} z+O\left(z^{2}\right)
\end{gathered}
$$

In these equations, $w_{o}$ and $u_{o}$ are the displacements at the middle surface $z=0$. As described by [2], the Kirchhoff hypothesis is comprised of two assumptions: 1) that normals to the undeformed middle surface remain straight and normal and 2) the length of these normals is preserved, i.e., radial deformation is constant through the wall thickness. The later condition dictates that $w(x, z)=w_{o}(x)$ so only the first term in Eq. (2b) is non-zero. The assumption that normals to the undeformed middle surface remain straight and normal can only be satisfied if the shear strain $\varepsilon_{z x}$ from Eq. (1) is zero. Application of this constraint to Eq. (2) reveals that:

$$
\left.\frac{\partial u}{\partial z}\right|_{z=0}=-\frac{\partial w_{o}}{\partial x}
$$

and the coefficients of all terms of quadratic and higher order in Eq. (2a) are zero. As a result, the displacement field that satisfies the Kirchhoff hypothesis is the following:

$$
\begin{aligned}
& w(x, z)=w_{o}(x) \\
& u(x, z)=u_{o}(x)-\frac{\partial w_{o}}{\partial x} z
\end{aligned}
$$

Upon substituting Eqs. (4) into Eqs. (1), the axial and hoop strains become:

$$
\begin{aligned}
\varepsilon_{x} & =\frac{\partial u_{o}}{\partial x}-\frac{\partial^{2} w_{o}}{\partial x^{2}} z \\
\varepsilon_{\theta} & =\frac{w_{o}}{a+z}=\frac{w_{o}}{a}-\frac{w_{o}}{a^{2}} z+O\left(z^{2}\right)
\end{aligned}
$$

In the latter of these equations, a Taylor series in $z$ has been applied. These two equations relate the strain at any point through the wall thickness of the tube to the displacements $u_{o}$ and $w_{o}$ of the middle surface. The strains at the outer and inner surfaces of the tube $(z= \pm h / 2)$ are then:

$$
\begin{aligned}
\varepsilon_{x} & =\frac{\partial u_{o}}{\partial x} \mp \frac{\partial^{2} w_{o}}{\partial x^{2}} \frac{h}{2} \\
\varepsilon_{\theta} & =\frac{w_{o}}{a}\left(1 \mp \frac{h}{2 a}\right)
\end{aligned}
$$

By assuming that radial stresses are small in comparison to hoop and axial stresses, Hooke's law can be applied to show that the stress resultants (i.e., the integrals of stresses through the wall thickness) in the axial and circumferential directions are given by [13]:

$$
\begin{aligned}
& N_{x}=\frac{E}{1-v^{2}} \int_{-h / 2}^{h / 2}\left(\varepsilon_{x}+v \varepsilon_{\theta}\right) d z \\
& N_{\theta}=\frac{E}{1-v^{2}} \int_{-h / 2}^{h / 2}\left(\varepsilon_{\theta}+v \varepsilon_{x}\right) d z
\end{aligned}
$$

Upon substituting the strain-displacement relations from Eq. 5, the stress resultants become:

$$
\begin{aligned}
& N_{x}=\frac{E h}{1-v^{2}}\left(\frac{\partial u_{o}}{\partial x}+v \frac{w_{o}}{a}\right) \\
& N_{\theta}=\frac{E h}{1-v^{2}}\left(\frac{w_{o}}{a}+v \frac{\partial u_{o}}{\partial x}\right)
\end{aligned}
$$


It is interesting to note that the strains in Eq. (6) have errors of order $O\left(h^{2}\right)$, while the errors in the stress resultants of Eq. (8) are of order $O\left(h^{3}\right)$. To demonstrate this, one could include terms up to quadratic order in Eq. (5) and find that they are identically equal to zero after the integration in Eq. (7). This result suggests that middle-surface strains, which are computed by applying conservation laws to the stress resultants, may be predicted more accurately than strains at the inner and outer surfaces of the tube.

\section{TYPES OF ERRORS IN SHELL MODELS}

For a tube subjected to any set of load conditions, the calculation of strains at the inner and outer surfaces consists of two steps. First, conservation laws are applied to the stress and moment resultants in order to determine the displacements $u_{o}$ and $w_{o}$ at the middle surface of the tube. Second, the strain-displacement relations of Eq. (6) are applied to calculate strains at the inner and outer surfaces. Each of these steps introduces errors, and one objective of this paper is to analyze and quantify them.

In the first part of this paper, we assume that the middlesurface displacements $w_{o}$ and $u_{o}$ are known a priori. This eliminates errors that would arise in the calculation of $u_{o}$ and $w_{o}$ and allows the errors due to the application of Eq. (6) alone to be analyzed. To evaluate these errors, a prescribed middle-surface displacement field is applied to both the shell model and a finite element model and the differences in outer-surface strain are analyzed.

The second part of this paper focuses on the accurate prediction of the middle-surface displacements $u_{o}$ and $w_{o}$. Emphasis is placed on predicting the behavior of thick-walled tubes that are loaded by shock or detonation waves. A dynamic shell model is developed and its predictions are compared with the results of finite element simulations.

\section{ERRORS DUE TO STRAIN-DISPLACEMENT RELA- TIONS}

This section considers the ability of strain-displacement relations such as those in Eq. (6) to accurately predict strains at the inner and outer surfaces of a tube. This is done by conducting finite element simulations of a section of pipe subjected to prescribed middle-surface displacements and then comparing the resulting outer-surface strains with those predicted by Eq. (6) using the same middle surface deformation.

For both the shell model and the finite element model, a sinusoidal deformation of the following form is imposed at the middle surface of the tube:

$$
w_{o}=\frac{\delta}{2}\left[1-\cos \left(\frac{2 \pi x}{\lambda}\right)\right]
$$

where $\lambda$ is the wavelength of the sinusoid. This deformation is zero at the ends of the tube and reaches a maximum radial displacement of $\delta$ at the center. Deformation of this form is motivated by the sinusoidal vibrations that are observed behind a shock or detonation wave [4], which will be considered in more detail in Sec. 5.

\subsection{Expectations from Shell Theory}

After substituting the deformation of Eq. (9) into Eq. (5b), the hoop strain evaluated at $z= \pm h / 2$ is found to be:

$$
\varepsilon_{\theta}=\frac{\delta}{2 a}\left(1 \mp \frac{h}{2 a}\right)\left[1-\cos \left(\frac{2 \pi x}{\lambda}\right)\right]
$$

and its maximum value is:

$$
\varepsilon_{\theta, \max }=\frac{\delta}{a}\left(1+\frac{h}{2 a}\right)
$$

This maximum occurs at the inner surface of the tube $(z=-h / 2)$ at an axial position of $\lambda / 2$, and its value does not depend on the wavelength of the deformation.

The axial strain in Eq. 5a consists of two terms. The first term is known as the membrane strain, and evaluation of this term requires a model for the axial displacement $u_{o}$. Since the deformation imposed here is motivated by the sequence of vibrations following a shock or detonation wave, it would be appropriate to choose an axial displacement model that is consistent with that scenario. One model that is frequently used is to assume that the axial stress behind the detonation wave is small, that is, $N_{x} \approx 0$. Referring to Eq. (8a), the assumption of zero axial stress implies that the membrane strain is given by:

$$
\varepsilon_{x, \text { membrane }} \equiv \frac{\partial u_{o}}{\partial x}=-v \frac{w_{o}}{a}
$$

Upon substituting the assumed displacement field from Eq. (9), the membrane strain becomes:

$$
\varepsilon_{x, \text { membrane }}=-\frac{v \delta}{2 a}\left[1-\cos \left(\frac{2 \pi x}{\lambda}\right)\right]
$$

This component of strain arises due to the Poisson coupling between radial and axial motion. When the tube expands radially, stretching of the tube is accompanied by a contraction in the axial direction, and the magnitude of this contraction is the axial membrane strain. By comparison of this equation with Eq. 10, it is seen that the membrane strain is always out of phase with the hoop strain. 
The second term in Eq. 5a is known as the bending strain, given by:

$$
\varepsilon_{x, b e n d} \equiv-\frac{\partial^{2} w_{o}}{\partial x^{2}} z
$$

Upon substituting the assumed deformation from Eq. (9) and evaluating at $z= \pm h / 2$, the bending strain becomes:

$$
\varepsilon_{x, \text { bend }}=\mp \pi^{2} \frac{h \delta}{\lambda^{2}} \cos \left(\frac{2 \pi x}{\lambda}\right)
$$

This component of the axial strain is the result of changes in curvature of the middle surface. At the outer surface of the tube, the bending strain is out of phase with the hoop strain from Eq. (10), while at the inner surface it is in phase.

After adding together the membrane and bending strains, the total axial strain evaluated at $z= \pm h / 2$ is:

$$
\varepsilon_{x}=-\frac{\delta v}{2 a}+\left(\frac{\delta v}{2 a} \mp \frac{\pi^{2} \delta h}{\lambda^{2}}\right) \cos \left(\frac{2 \pi x}{\lambda}\right)
$$

At the inner surface of the tube, both terms in the coefficient of the cosine are positive, and hence they are additive. As a result, the maximum axial strain occurs when the cosine takes on a value of -1 , which produces a maximum strain of:

$$
\varepsilon_{x, \max }=-\frac{v \delta}{a}-\pi^{2} \frac{\delta h}{\lambda^{2}} \quad \text { (Inner Surface) }
$$

At the outer surface of the tube, however, the terms involved in the coefficient of the cosine are of opposite sign and cancellation occurs. At a critical wavelength $\lambda_{c r}$, these two terms cancel out entirely leaving an axial strain with no spatial variation. The critical wavelength at which this occurs is:

$$
\frac{\lambda_{c r}}{h}=\pi \sqrt{\frac{2 a}{v h}}
$$

The axial strain in Eq. (16) can only reach positive values if the wavelength is smaller than this critical value. Furthermore, if $\lambda>\lambda_{c r}$, then the outer surface axial strain is out of phase with the hoop strain, while for smaller values of $\lambda$ it is in phase.

\subsection{Static Finite Element Model}

Static finite element simulations were conducted using the program FEAPpv [14], discussed in Chap. 19 of [15]. The pipe

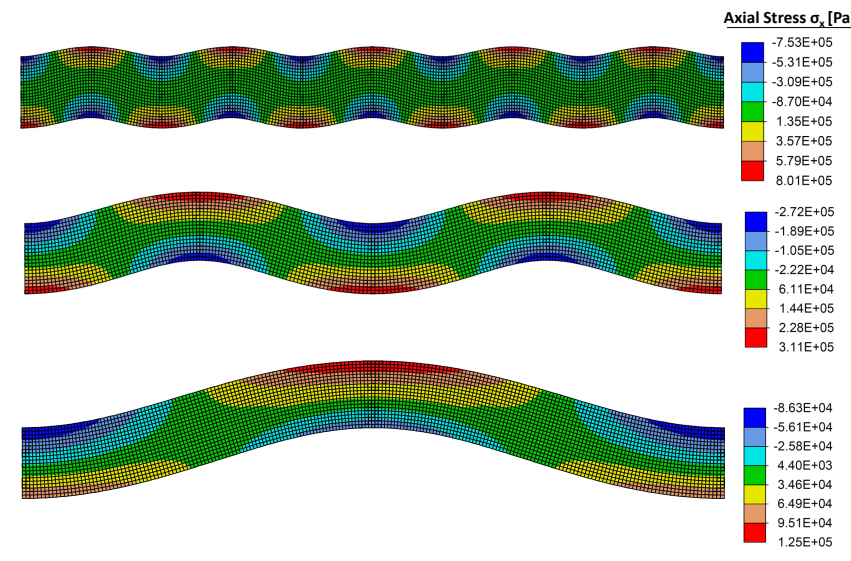

FIGURE 2. Contours of axial stress $\sigma_{x}$ for a tube with $a / h=7$ and three different values of $\lambda / h$. These values are top: $\lambda / h=2$; middle: $\lambda / h=5$; bottom: $\lambda / h=10$. In all three cases, $E=193 \mathrm{GPa}, v=0.29$, $R_{i}=26.25 \mathrm{~mm}$, and the maximum displacement of the middle surface is $\delta=20 \mu \mathrm{m}$.

was modeled using 4-node, axisymmetric quadrilateral elements. The number of nodes through the wall thickness was always 20 , while the number of nodes along the axis was varied as the tube length was changed in order to preserve an element aspect ratio of 1.0. The pipe was assumed to be made from an isotropic material with $E=193 \mathrm{GPa}$ and $v=0.29$, which are representative of 304 stainless steel. The inner radius of the pipe was fixed at $26.25 \mathrm{~mm}$, which is the nominal value for 2 inch schedule 40 pipe, and the outer radius was varied to model pipes of several wall thicknesses. Three different pipe sizes with $a / h=20,7$, and 4 were analyzed. These values of $a / h$ are close to those for 2 inch pipe of schedules 5, 40, and 120 .

The same sinusoidal deformation from Eq. (9) was applied at the middle surface of the tube. Since the objective is to model the series of vibrations that occurs behind a detonation wave, periodic boundary conditions were imposed at the ends of the pipe. Several examples of axial stress fields for various values of $\lambda / h$ are shown in Fig. 2. These plots demonstrate the increasing stresses (for fixed radial displacement) at the inner and outer surfaces of the tube as the wavelength of deformation approaches the tube wall thickness.

\subsection{Comparison between Shell Model and FEM}

Since the boundary conditions chosen for the finite element model are periodic, $\partial u_{o} / \partial x=0$ is imposed at the boundaries and hence the membrane strain is zero. As a result, the axial strains can be computed using only the bending contribution, given in Eq. (15). These predictions are compared with finite element 


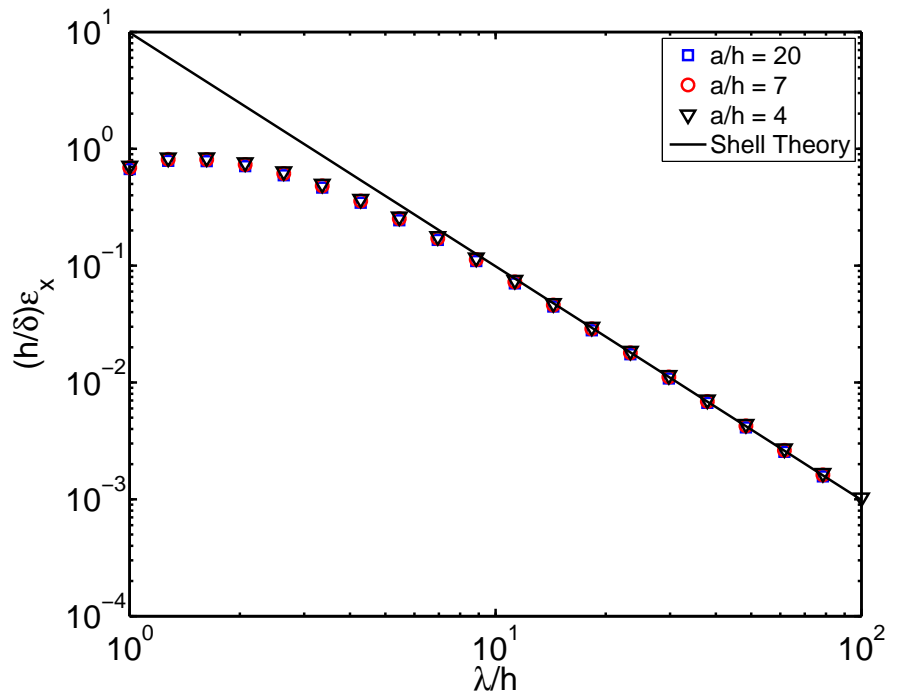

FIGURE 3. Variation of maximum axial strain (same for both inner and outer surfaces of tube) with dimensionless wavelength of deformation. Solid line: shell theory. Markers: finite element analysis.

results in Fig. 3. Equation (15) suggests that if the wavelength $\lambda$ is normalized by the wall thickness $h$ and the axial strains are scaled by $h / \delta$, the strains for different wall thicknesses should collapse onto a single curve. As shown in the Fig. 3, this is the case, even for small wavelengths where the predictions of the finite element model deviate from those of the shell model. This scaling demonstrates that the axial strains do not depend strongly on the mean radius $a$ of the tube.

For large wavelengths $\lambda / h$, the finite element predictions are asymptotic to those from shell theory. In this limit, the slope of -2 on the log-scale plot confirms the inverse quadratic relationship between the outer-surface axial strain and the wavelength of the deformation that was predicted by Eq. (15). At small wavelengths, the predictions of the finite element model deviate substantially from this trend. Nevertheless, for wavelengths having $\lambda / h>10$, the relative error between the finite element and shell models is less than $10 \%$.

Figure 4 shows the ratio of axial strain (Eq. 15) to hoop strain (Eq. 11) as a function of axial wavelength. For long wavelengths the axial strain is negligible in comparison to the hoop strain, but for small wavelengths the axial strains can be of similar magnitude or larger than the hoop strains. In Section 5 it is demonstrated that for tubes loaded by shock waves, $\lambda / h$ can take on values in the range of 2-10 and axial strains can have similar amplitudes to the hoop strains. This demonstrates that in designing piping systems to withstand detonation loads, it is often necessary to consider the bi-axial state of stress at the inner and outer surfaces of the tube rather than assuming that hoop component is dominant.

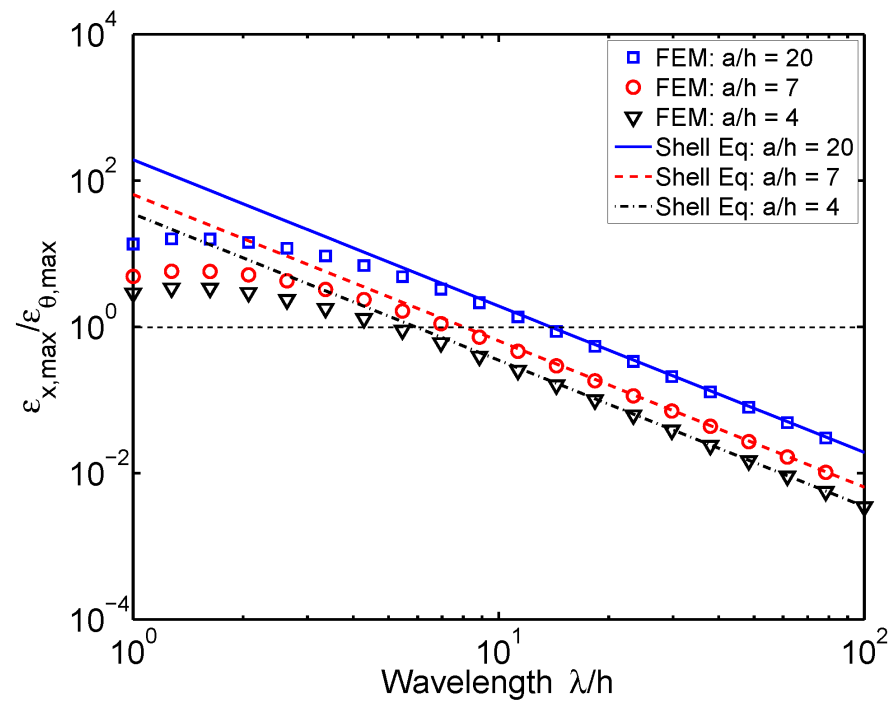

FIGURE 4. Ratio of maximum axial strain to maximum hoop strain as a function of wavelength for several tube sizes. Axial strains are greater than hoop strains above horizontal line.

\section{PERFORMANCE OF DYNAMIC SHELL EQUATIONS}

We have now shown that for a given set of middle-surface displacements $w_{o}$ and $u_{o}$, the shell equations adequately predict outer-surface strains as long as the wavelength of the deformation is greater than about $\lambda / h \sim 10$. We now consider whether or not the middle-surface actually deforms into such small wavelengths under practical conditions and investigate whether or not transient shell models are capable of accurately predicting the middle-surface displacements for thick tubes. This is done by comparing the predictions of a transient shell model with those of highly resolve finite element simulations.

\subsection{Shell Models of Dynamic Tube Response}

In the sections that follow, an axisymmetric dynamic shell model based on the work of Tang [11] is described and a solution technique is presented for solving the initial value problem of a shock wave pressure load traveling along the tube at a fixed velocity. The chosen shell model includes the effects of transverse shear deformation and rotary inertia of the pipe, both of which are important when modeling thick-walled tubes and high-speed loads $[16,17]$.

5.1.1 Equations of Motion Following the approach of [11], the dynamic shell equations for a pipe under a pressure 
load $p$ are given by:

$$
\begin{aligned}
\rho h \frac{\partial^{2} u_{o}}{\partial t^{2}} & =\frac{\partial N_{x}}{\partial x} \\
\rho h \frac{\partial^{2} w_{o}}{\partial t^{2}} & =\frac{\partial Q_{x}}{\partial x}-\frac{N_{\theta}}{a}+p \\
\frac{\rho h^{3}}{12} \frac{\partial^{2} \psi_{x}}{\partial t^{2}} & =\frac{\partial M_{x}}{\partial x}-Q_{x}
\end{aligned}
$$

Here $u_{o}, w_{o}$, and $\psi_{x}$ are the axial, radial, and angular displacements of the middle surface, and $\rho, h$, and $a$ are the tube's density, wall thickness, and mean radius. The axial and circumferential stress resultants, denoted $N_{x}$ and $N_{\theta}$, and the shear and moment resultants, $Q_{x}$ and $M_{x}$, are given by

$$
\begin{aligned}
N_{x} & =\frac{E h}{1-v^{2}}\left[\frac{\partial u_{o}}{\partial x}+v \frac{w_{o}}{a}\right] \\
N_{\theta} & =\frac{E h}{1-v^{2}}\left[\frac{w_{o}}{a}+v \frac{\partial u_{o}}{\partial x}\right] \\
Q_{x} & =\kappa G h\left[\psi_{x}+\frac{\partial w_{o}}{\partial x}\right] \\
M_{x} & =\frac{E h^{3}}{12\left(1-v^{2}\right)} \frac{\partial \psi_{x}}{\partial x}
\end{aligned}
$$

The stress resultants above are the same as those obtained in Eq. (8), so the present formulation is consistent with the strains presented in Eq. (6).

Using the approach of [11], the radial displacement $w_{o}$ of the middle surface is decomposed into a component due to bending, $w_{b}$, and a component due to shear, $w_{s}$, such that $w_{b}+w_{s}=w_{o}$. The bending contribution satisfies (by definition) the relation $\partial w_{b} / \partial x=-\psi_{x}$. Here a slight departure from the formulation in Sec. 2 is introduced by the inclusion of transverse shear deformation, $w_{s}$. Since the shear deformation $w_{s}$ does not change the axial curvature of the middle surface, it has no effect on the axial strains at distances $z$ away from the middle surface. Thus to properly calculate the axial strains at the inner and outer surfaces of the tube, only the part of the deformation due to bending, $w_{b}$, should be used to evaluate $w_{o}$ in Eq. (5a). However, hoop strains do not involve the change in axial curvature of the middle surface, so the total deformation $w_{o}=w_{s}+w_{b}$ should be used to evaluate the hoop strains in Eq. (5b).

It is convenient to develop and solve the shell model in terms of the following dimensionless parameters:

$$
\begin{array}{rlrl}
W_{b} & =\frac{w_{b}}{h} & W_{s} & =\frac{w_{s}}{h} \\
U & =\frac{u_{o}}{h} & \beta^{2} & =\frac{h^{2}}{12 a^{2}} \\
X & =\frac{\sqrt{12}}{h} x & T & =\frac{\sqrt{12} v_{d}}{h} t \\
\delta^{2} & =\frac{\kappa G\left(1-v^{2}\right)}{E} & P & =\frac{1-v^{2}}{12 E}
\end{array}
$$

The parameter $v_{d}$ is the shell dilatational wave velocity, given by $v_{d}=\sqrt{E /\left(1-v^{2}\right) \rho}$, and $\kappa$ is the shear correction factor, assumed here to have a value of $5 / 6$ as proposed by [16]. Eliminating the stress resultants from Eqs. (19-20) and expressing the result in terms of the dimensionless parameters (21) gives:

$$
\begin{aligned}
\frac{\partial^{2} U}{\partial T^{2}}-\frac{\partial^{2} U}{\partial X^{2}}-v \beta\left(\frac{\partial W_{b}}{\partial X}+\frac{\partial W_{s}}{\partial X}\right) & =0 \\
\frac{\partial^{2}}{\partial T^{2}}\left(W_{s}+W_{b}\right)-\delta^{2} \frac{\partial^{2} W_{s}}{\partial X^{2}}+\beta^{2}\left(W_{s}+W_{b}\right)+v \beta \frac{\partial U}{\partial X} & =P \\
\frac{\partial^{2} W_{b}}{\partial X^{2}}-\frac{\partial^{2} W_{b}}{\partial T^{2}}+\delta^{2} W_{s} & =0
\end{aligned}
$$

The boundary conditions chosen for this analysis are the following:

$$
\begin{aligned}
W(0, T)=W(L, T) & =0 \\
\left.\frac{\partial^{2} W}{\partial X^{2}}\right|_{X=0, L} & =0 \\
\left.\frac{\partial U}{\partial X}\right|_{X=0, L} & =0
\end{aligned}
$$

The radial boundary conditions are those of a simply-supported tube. Referring to Eq. (20a), the axial boundary condition is one of zero axial stress $\left(N_{x}=0\right)$ corresponding to a tube with ends that can deform freely in the axial direction.

A Fourier series expansion in the $X$ coordinate which satis- 
fies these boundary conditions is taken in the following form:

$$
\begin{aligned}
U & =\sum_{n=1}^{\infty} U_{n} \cos (k X) \\
W_{s} & =\sum_{n=1}^{\infty} S_{n} \sin (k X) \\
W_{b} & =\sum_{n=1}^{\infty} B_{n} \sin (k X) \\
P & =\sum_{n=1}^{\infty} P_{n} \sin (k X)
\end{aligned}
$$

where $k=n \pi / L$. Inserting these series into Eqs. (22) reduces the system to a set of three linear, second order ODEs in time:

$$
\begin{aligned}
& \ddot{U}_{n}=k v \beta\left(B_{n}+S_{n}\right)-k^{2} U_{n} \\
& \ddot{S}_{n}=B_{n}\left(k^{2}-\beta^{2}\right)-S_{n}\left[\delta^{2}\left(1+k^{2}\right)+\beta^{2}\right] \\
& +k v \beta U_{n}+P_{n}(T) \\
& \ddot{B}_{n}=\delta^{2} S_{n}-k^{2} B_{n}
\end{aligned}
$$

These can be expressed as a first order linear system:

$$
\frac{\partial \mathbf{X}}{\partial t}=\tilde{\mathbf{A}} \mathbf{X}+\mathbf{F} P_{n}(T)
$$

where

$$
\begin{aligned}
\mathbf{X} & =\left[U_{n}, B_{n}, S_{n}, \dot{U}_{n}, \dot{B}_{n}, \dot{S}_{n}\right]^{T} \\
\mathbf{F} & =[0,0,0,0,0,1]^{T}
\end{aligned}
$$

and

$$
\tilde{\mathbf{A}}=\left[\begin{array}{cccccc}
0 & 0 & 0 & 1 & 0 & 0 \\
0 & 0 & 0 & 0 & 1 & 0 \\
0 & 0 & 0 & 0 & 0 & 1 \\
-k^{2} & k v \beta & k v \beta & 0 & 0 & 0 \\
0 & -k^{2} & \delta^{2} & 0 & 0 & 0 \\
k v \beta & \left(k^{2}-\beta^{2}\right)-\left(\beta^{2}+\delta^{2}+k^{2} \delta^{2}\right) & 0 & 0 & 0
\end{array}\right]
$$

The form of the forcing term $P_{n}(T)$ is found by assuming that the pressure load is a step function of amplitude $P_{o}$ traveling along the tube at a dimensionless velocity $V=v / v_{d}$

$$
P(x, t)=P_{o} H(V T-X)=\sum_{n=0}^{\infty} P_{n}(t) \sin k X
$$

where $H$ is the Heaviside step function. By integrating over the domain and using the orthogonality of the eigenfunctions, the coefficients $P_{n}$ are determined to be:

$$
P_{n}(t)=\frac{2 P_{o}}{n \pi}[1-\cos (k V T)]
$$

In this analysis, the eigenvalues of the matrix $\tilde{\mathbf{A}}$ always consisted of six distinct values which were purely imaginary. Although it has not been proven that this is always the case, the eigenvalues differ only slightly from those in shell formulations without shear deformation and rotary inertia, for which it can be shown that the eigenvalues are always distinct and purely imaginary. Further support for the assumption of distinct imaginary eigenvalues is obtained on physical grounds: If any eigenvalue has a non-zero real part, then the tube's response will grow exponentially with time, even if no pressure load is applied. Likewise, a repeated eigenvalue will produce unbounded linear growth of deformation with time even when the system is unforced. Such behavior is non-physical in the absence of applied loads since no mechanism for the addition or depletion of energy is available. When the system is forced (for instance, by a traveling pressure wave), unbounded growth may occur due to resonance between the tube vibration and the applied load, but this does not involve complex or repeated eigenvalues.

Dispersion curves for three tube thickness of $a / h=4,7$, and 20 are shown in Fig. 5. For real wavenumbers the velocity spectrum is composed of three branches, with branch 1 consisting of coupled radial and axial motion, branch 2 being predominantly axial, and branch 3 being predominantly rotary. For practical cases involving detonations or shock waves in metal tubes, the velocities are small enough that the first branch plays the most important role in the response. The dispersion curves can be used to estimate the most prominent wavelengths in the response: these are the intersection points between the load speed and the dispersion curve, which correspond to steady-state solutions to the shell equations $[3,11]$. As discussed by Tang, these steadystate solutions exhibit a resonance at the critical velocity $v_{c o}$ corresponding to the minimum of the first branch of the dispersion curve [11]. Our simulations show that except near this velocity, the tube response rapidly approaches these steady-state solutions after the load has traveled several flexural wavelengths past in incident end of the tube. In the sections that follow, loads traveling at a constant speed of $1800 \mathrm{~m} / \mathrm{s}$ are applied to the tube, which corresponds to the horizontal line at 0.35 in Fig. 5.

5.1.2 Solution Technique The homogeneous solution $\mathbf{X}_{\mathbf{h}}$ to Eq. 26 is obtained using the matrix exponential:

$$
\mathbf{X}_{\mathbf{h}}(t)=e^{\tilde{\mathbf{A}} t} \mathbf{C}_{\mathbf{h}}
$$




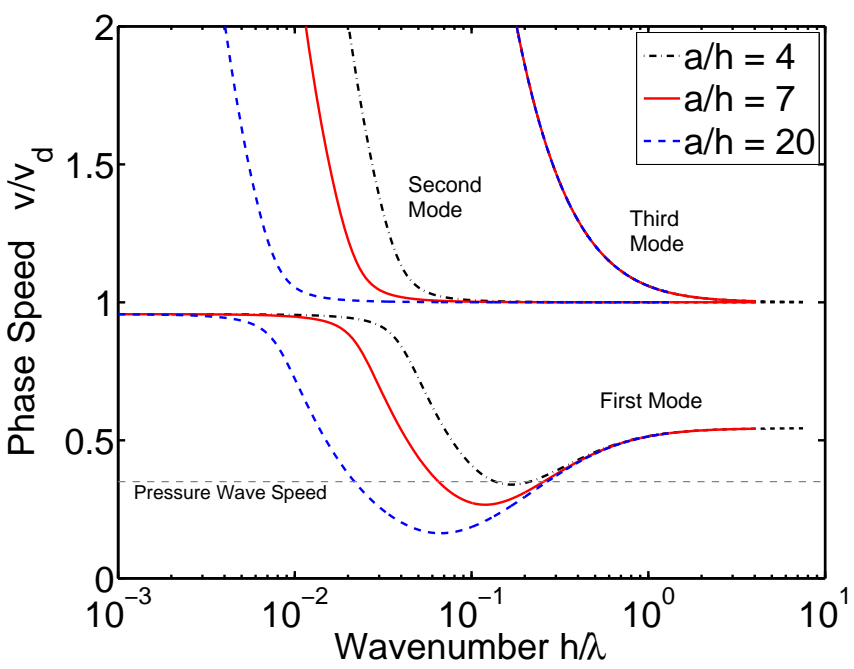

FIGURE 5. Dispersion curves for tubes with $a / h=4,7$, and 20 and $v_{d}=5120 \mathrm{~m} / \mathrm{s}$.

where $\mathbf{C}_{\mathbf{h}}$ is a constant vector which will be determined after finding the particular solution and applying initial conditions. To find the particular solution $\mathbf{X}_{\mathbf{p}}$, the method of undetermined coefficients is used with the solution assumed to be of the following form:

$$
\mathbf{X}_{\mathbf{p}}=\mathbf{C}_{\mathbf{o}}+\mathbf{C}_{\mathbf{1}} \cos (k V t)+\mathbf{C}_{\mathbf{2}} \sin (k V t)
$$

This form of particular solution is valid as long as $k V$ is not an eigenvalue of $\tilde{\mathbf{A}}$. If $k V$ is an eigenvalue, then additional terms are needed in the particular solution; however, in a numerical calculation the value of $k V$ is usually not exactly equal to an eigenvalue, so it is not necessary to include these additional terms. Upon substituting this particular solution into Eq. 26 and equating the coefficients of constants, sines, and cosines, the following set of matrix equations is obtained:

$$
\begin{array}{r}
\mathbf{C}_{\mathbf{1}} k V+\tilde{\mathbf{A}} \mathbf{C}_{\mathbf{2}}=0 \\
\tilde{\mathbf{A}} \mathbf{C}_{\mathbf{1}}-\mathbf{C}_{\mathbf{2}} k V=\mathbf{F}\left(\frac{2 P_{o}}{n \pi}\right) \\
\tilde{\mathbf{A}} \mathbf{C}_{\mathbf{o}}=\frac{2 P_{o}}{n \pi} \mathbf{F}
\end{array}
$$

The first two of these equations can be expressed in block-matrix form as follows:

$$
\left[\begin{array}{cc}
k V \mathbb{I} & \tilde{\mathbf{A}} \\
\tilde{\mathbf{A}} & k V \mathbb{I}
\end{array}\right]\left(\begin{array}{l}
\mathbf{C}_{\mathbf{1}} \\
\mathbf{C}_{\mathbf{2}}
\end{array}\right)=\left(\begin{array}{c}
\mathbf{0} \\
\mathbf{F}
\end{array}\right) \frac{2 P_{o}}{n \pi}
$$

where $\mathbb{I}$ is the $2 \times 2$ identity matrix and $\mathbf{0}$ is a $2 \times 1$ vector of zeros. Inverting ${ }^{1}$ and solving gives:

$$
\begin{aligned}
\left(\begin{array}{l}
\mathbf{C}_{\mathbf{1}} \\
\mathbf{C}_{\mathbf{2}}
\end{array}\right) & =\left(\begin{array}{l}
\mathbf{0} \\
\mathbf{F}
\end{array}\right)\left[\begin{array}{cc}
k V \mathbb{I} & \tilde{\mathbf{A}} \\
\tilde{\mathbf{A}} & k V \mathbb{I}
\end{array}\right]^{-1} \frac{2 P_{o}}{n \pi} \\
\mathbf{C}_{\mathbf{0}} & =\frac{2 P_{o}}{n \pi} \mathbf{F} \tilde{\mathbf{A}}^{-1}
\end{aligned}
$$

The particular solution is now completely determined. Together with the homogeneous solution from Eq. (29), the general solution of the equations of motion (Eq. 26) is given by:

$$
\mathbf{X}(t)=e^{\tilde{\mathbf{A}} t} \mathbf{C}_{\mathbf{h}}+\mathbf{C}_{\mathbf{o}}+\mathbf{C}_{\mathbf{1}} \cos k V t+\mathbf{C}_{\mathbf{2}} \sin k V t
$$

An appropriate initial condition is that all displacements and velocities are zero (i.e., $\mathbf{X}=0$ ) when $t=0$. Since the matrix exponential of a zero matrix is the identity matrix, the initial condition leaves:

$$
\mathbf{X}(0)=0=\mathbf{C}_{\mathbf{h}}+\mathbf{C}_{\mathbf{o}}+\mathbf{C}_{\mathbf{1}} \rightarrow \mathbf{C}_{\mathbf{h}}=-\mathbf{C}_{\mathbf{1}}-\mathbf{C}_{\mathbf{o}}
$$

All parameters of the general solution (Eq. 35) are now known. This solution can now be computed for each value of $n$, and the coefficients $B_{n}, S_{n}$, and $U_{n}$ can be extracted from the vector $\mathbf{X}$. The middle surface displacements are then evaluated from the summations in Eq. (24), and strains are computed using Eq. (6). As discussed previously, only the bending component of radial displacement $w_{b}$ is used in Eq. (5a) while the total radial displacement $w_{b}+w_{s}$ is used in Eq. (5b).

The number of terms required in the Fourier series depends on the length of the tube and the speed of the applied load. For example, if the length of the tube is doubled, the number of terms must be doubled in order to resolve the same maximum wavenumber. The largest wavenumber that must be resolved can be estimated from the dispersion curves (Fig. 5) by finding the intersection points between the load velocity and the dispersion curves.

\subsection{Dynamic Finite Element Modeling}

To analyze the performance of the above shell model, finite element simulations were conducted using the commercial software package LS-DYNA [18]. The pipe was modeled using axisymmetric, quadrilateral elements with 35 nodes through the tube wall, and the number of nodes along the tube axis was chosen by setting the aspect ratio of the elements equal to 1.0.

\footnotetext{
${ }^{1}$ The matrix is invertible as long as the assumed particular solution Eq. 30 is not also a solution to the homogeneous equation.
} 
For the results presented in this paper, these conditions correspond to 130-150 elements per axial wavelength for the shortest waves observed in the response. At this resolution the results are well-converged; however, no attempt was made to determine the coarsest mesh that is sufficiently resolved to accurately capture the smallest wavelengths.

As was done in the static analysis of Sec. 4, the pipe was modeled as elastic and isotropic with an $E=193 \mathrm{GPa}, v=0.29$, and $\rho=8040 \mathrm{~kg}-\mathrm{m}^{-3}$ (nominal values for 304 stainless steel). A $2 \mathrm{~m}$ long segment of pipe was modeled with inner radius of $26.25 \mathrm{~mm}$ and wall thicknesses of 7.5, 4.0, and $1.3 \mathrm{~mm}$. These give values $a / h$ of 4,7 , and 20, which are similar to those for 2-inch pipe of schedules 5, 40, and 120 . The load applied to the tube was taken to be a shock wave of amplitude $p_{o}=2.5 \mathrm{MPa}$ traveling along the tube with a speed of $1800 \mathrm{~m} / \mathrm{s}$. These conditions are representative of detonations in hydrocarbon-air mixtures at atmospheric initial pressure [19].

The boundary conditions for the finite element model were chosen to mirror those of the dynamic shell model as closely as possible. At the ends of the tube, only the nodes lying on the middle surface of the pipe were constrained radially so that the ends of the pipe are free to rotate. This matches the boundary condition in the shell model from Eq. (23), where the radial displacements are zero but the slope $\partial w / \partial x$ at the ends of the pipe can be non-zero. In order to match the axial boundary condition in Eq. (23), the ideal constraint would be $\partial u / \partial x=0$ at both ends of the tube, which is satisfied if the ends of the tube are free of axial forces. However, such a boundary condition is not admissible in FEM since the tube must be anchored axially in at least one place, so the boundary condition $u(L, t)=0$ was also applied. As a result, the solutions from the finite element simulation are only expected to match the shell model until the wave system that develops first encounters the end of the pipe $x=L$.

\subsection{Comparison of Transient Shell Model with FEM}

Before making comparisons between the different tube sizes, it is worth noting that the qualitative features of the tube response depend strongly on the ratio of the applied load speed $v$ to the "first critical velocity," $v_{c o}$. The behavior of tubes near this critical velocity has been studied by numerous authors $[4,11,12,20]$ who have found that a resonance between the traveling load and the speed of flexural waves results in highly amplified displacements, stresses, and strains. This critical velocity is the minimum phase speed of sinusoidal waves in the tube, and hence is located at the minimum of the lowest branch of the dispersion curve (see Fig. 5). As indicated in that figure, the critical speed increases significantly as the tube is made thicker. In the results that follow, the speed of the load is held fixed at $1800 \mathrm{~m} / \mathrm{s}$ while the thickness of the tube is increased. As a result, for thicker tubes the load speed is closer to the critical velocity.

Strain traces for three different tube sizes are compared in

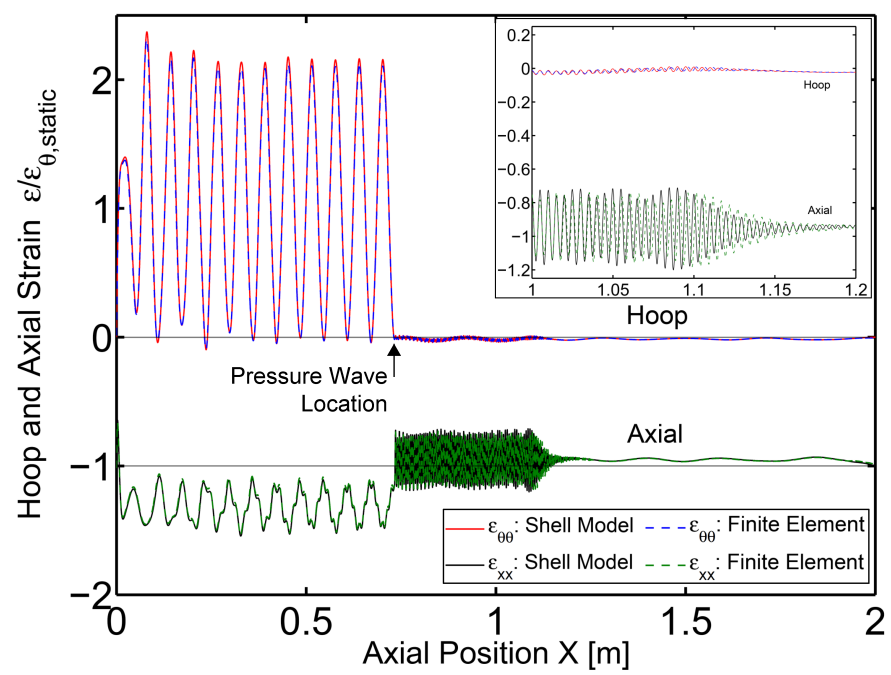

FIGURE 6. Comparison finite element and shell model predictions of hoop and axial strains at the outer surface of a pipe. The pipe has mean radius $a=26.9 \mathrm{~mm}$ and wall thickness $h=1.34 \mathrm{~mm}(a / h=20)$. Load velocity is $v=1800 \mathrm{~m} / \mathrm{s}$, which corresponds to $v / v_{d}=0.35$ and $v / v_{c o}=2.2$. Axial strains have been offset by -1 for clarity. Inset plot magnifies the high frequency waves ahead of the load.

Figs. 6-8. To facilitate comparisons between the different wall thicknesses, all strains have been normalized by the static hoop strain that would occur at the applied pressure of $p_{o}=2.5 \mathrm{MPa}$ :

$$
\varepsilon_{\theta, \text { static }}=\frac{p_{o} a\left(1-v^{2}\right)}{E h}
$$

Figure 6 compares hoop and axial strains for a rather thin tube with $a / h=20$. The position of the pressure wave corresponds to the large jump in hoop strain at about $0.75 \mathrm{~m}$. The response of the tube consists primarily of two wavelengths: ahead of the pressure load the vibrations have a very short wavelength of about $\lambda=5.0 \mathrm{~mm}(\lambda / h=3.7)$ while behind the pressure load the wavelength is $62 \mathrm{~mm}(\lambda / h=46)$. These two wavelengths correspond to the two intersection points between the load speed and the dispersion curve, as shown in Fig. 5. The predominance of these two frequencies has also been verified experimentally by Beltman et al. [3]. Within the low-frequency vibrations behind the load, the peak hoop and axial strains from the finite element and shell models differ only by $2-3 \%$, which is expected since the wavelength of these is vibrations fairly large compared to the wall thickness.

The high-frequency vibrations ahead of the pressure load (magnified in the inset plot) are much more prominent in the axial strains than in the hoop strains. Although the frequency of these waves is well-predicted, the peak strains from the shell model are $10-30 \%$ greater than those from finite elements. This 


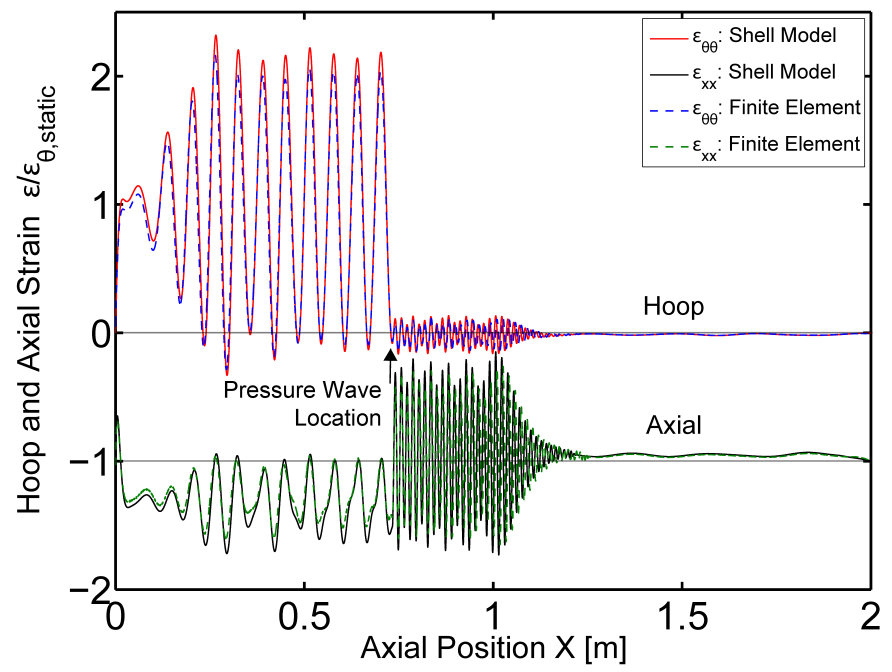

FIGURE 7. Comparison finite element and shell model predictions of hoop and axial strains at the outer surface of a pipe. The pipe has mean radius $a=28.3 \mathrm{~mm}$ and wall thickness $h=4.0 \mathrm{~mm}(a / h=7)$. Load velocity is $v=1800 \mathrm{~m} / \mathrm{s}$, which corresponds to $v / v_{d}=0.35$ and $v / v_{c o}=1.32$. Axial strains have been offset by -1 for clarity.

result is in agreement with the expectations from Fig. 3, where significant errors are predicted for the present value of $\lambda / h=3.7$.

The leading edge of the high-frequency wave packet travels at the shear wave speed, $v_{s}=\sqrt{\kappa G / \rho} \sim 2800 \mathrm{~m} / \mathrm{s}$, which is faster than the load speed, so this packet increases in width as time progresses. Ahead of the shear waves is a small amplitude bar wave, primarily an axial wave, which travels at the dilatation wave velocity $v_{d}=\sqrt{E / \rho\left(1-v^{2}\right)}$. At the time shown in Fig. 6, the bar wave is just reaching the end of the pipe.

The results in Fig. 6 demonstrate that for very thin tubes, the predictions of the transient shell models are virtually indistinguishable from those of finite element simulations, which is expected given the relatively long axial wavelength of the vibrations. Only in the high-frequency shear waves ahead of the load do the predictions of the shell model and the finite elements differ appreciably.

Figure 7 shows the hoop and axial strains for a slightly thicker tube with $a / h=7$. Again very good agreement is observed; however, the peak hoop strains predicted by the shell model are about $7 \%$ greater than those reported in the FEM. The low-frequency flexural waves behind the load again have a wavelength of about $62 \mathrm{~mm}$ which corresponds to $\lambda / h=16$, while the high-frequency waves ahead of the load have $\lambda=15.7 \mathrm{~mm}$, or $\lambda / h=4$.

Figure 8 shows hoop and axial strains at the outer surface of an even thicker-walled tube with $a / h=4$. In this case, the load speed of $1800 \mathrm{~m} / \mathrm{s}$ is only about 1.04 times the first critical velocity $v_{c o}$, so strains are highly amplified relative to the static

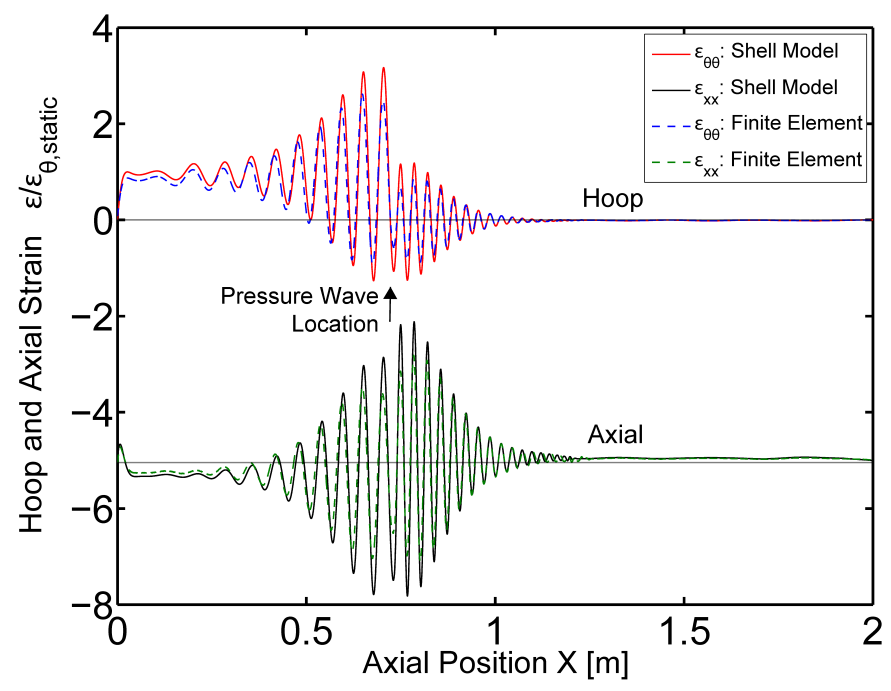

FIGURE 8. Comparison finite element and shell model predictions of hoop and axial strains at the outer surface of a pipe. The pipe has mean radius $a=30.0 \mathrm{~mm}$ and wall thickness $h=7.5 \mathrm{~mm}(a / h=4)$. Load velocity is $v=1800 \mathrm{~m} / \mathrm{s}$, which corresponds to $v / v_{d}=0.35$ and $v / v_{c o}=1.04$. Axial strains have been offset by -5 for clarity.

values. As shown in Fig. 5, the nearness of the load speed to the critical velocity causes the wavelengths ahead of the pressure wave $(35 \mathrm{~mm})$ and behind it $(56 \mathrm{~mm})$ to be very close to one another. These wavelengths correspond to $\lambda / h=7.5$ and 4.7 , respectively. This behavior has also been observed in experiments by Beltman et al., who recorded hoop strain traces that look much like those in Fig. 8.

In Fig. 8 the wavelengths of the deformation are wellpredicted by the shell model, but the peak strains are in error by about $20 \%$ for the hoop strain and $30 \%$ for the longitudinal strain, with the strains from FEM being lower than those from the shell model. It should be noted that similar errors are observed in the middle surface displacements, so in this case the shell model's errors at the outer surface of the tube cannot be attributed to the strain-displacement relations alone. It is also worth noting that the axial and hoop strains have about the same amplitude, which demonstrates the importance of including the biaxial state of stress or strain in design calculations.

The differences in the qualitative features of the strain traces in Figs. 6-8 are primarily due to the changes in load velocity relative to the first critical velocity $v_{c o}$. Although the load velocity was held fixed at $v=1800 \mathrm{~m} / \mathrm{s}$ for all three figures, the critical velocities increased as the tube was made thicker, resulting in smaller values of $v / v_{c o}$ for the thicker tubes. By comparing Figs. 6-8, it can be seen that the frequency of the longer wavelength vibrations behind the shock wave does not depend strongly on the wall thickness of the tube. In fact, this frequency 


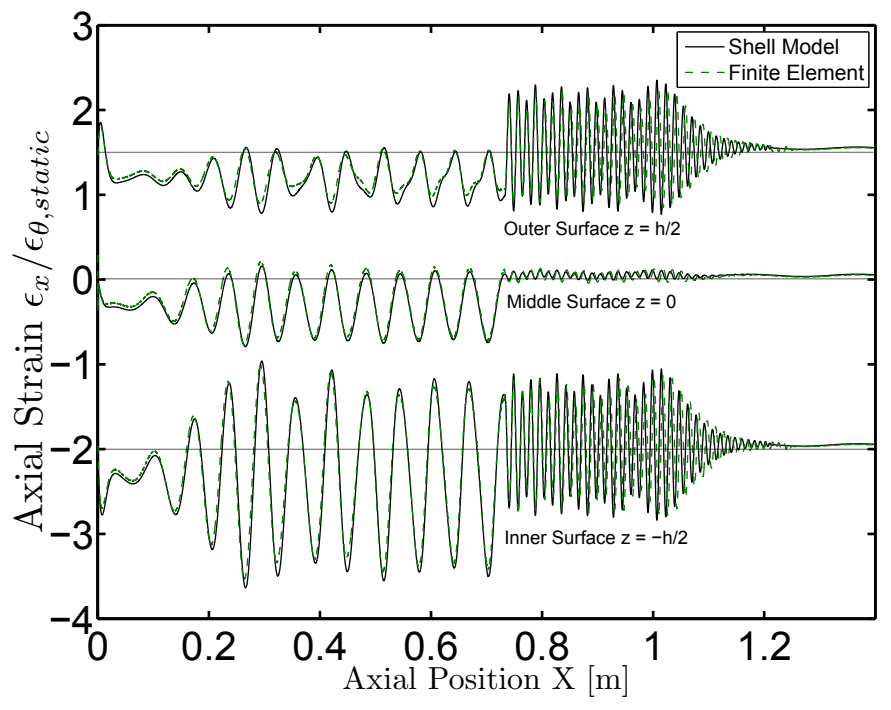

FIGURE 9. Comparison of axial strains at the inner, middle, and outer surfaces of a pipe. The pipe has mean radius $a=28.3 \mathrm{~mm}$ and wall thickness $h=4.0 \mathrm{~mm}(a / h=7)$. Load velocity is $v=1800 \mathrm{~m} / \mathrm{s}$, which corresponds to $v / v_{d}=0.35$ and $v / v_{c o}=1.32$. The strains at the inner and outer surfaces of the tube have been offset by -2 and +1.5 for clarity.

is usually close to that of an infinitely long tube [21]:

$$
f_{n}=\frac{1}{2 \pi a} \sqrt{\frac{E}{\rho\left(1-v^{2}\right)}}
$$

For metal pipes in 2-6 inch sizes, this frequency is on the order of $10-30 \mathrm{kHz}$, and for detonations in the range of $1500-2500 \mathrm{~m} / \mathrm{s}$, the associated wavelengths are $5-15 \mathrm{~cm}$. Such frequencies and wavelengths usually are not difficult to measure in experiment using ordinary strain gauges or predict in finite element models. In contrast, the wavelengths of the high frequency vibrations in the precursor wave ahead of the pressure load fall in the range of 1-10 times the wall thickness of the tube. As a result, these wavelengths are often in the range of $1-10 \mathrm{~mm}$ and have frequencies of $300 \mathrm{kHz}-3 \mathrm{MHz}$. Such small wavelengths and high frequencies are very difficult to measure experimentally and require high spatial and temporal resolution to predict correctly in finite element models.

\subsection{Variation of Strain through Wall Thickness}

So far, only the strains at the outer surfaces of the tube have been considered. However, often the axial strains at the inner surface are even larger than those at the outer surface. To illustrate this, axial strains at the inner, middle, and outer surfaces of a tube with $a / h=7$ are plotted in Fig. 9. At the middle surface of the tube, strains are due only to stretching of the tube and bending

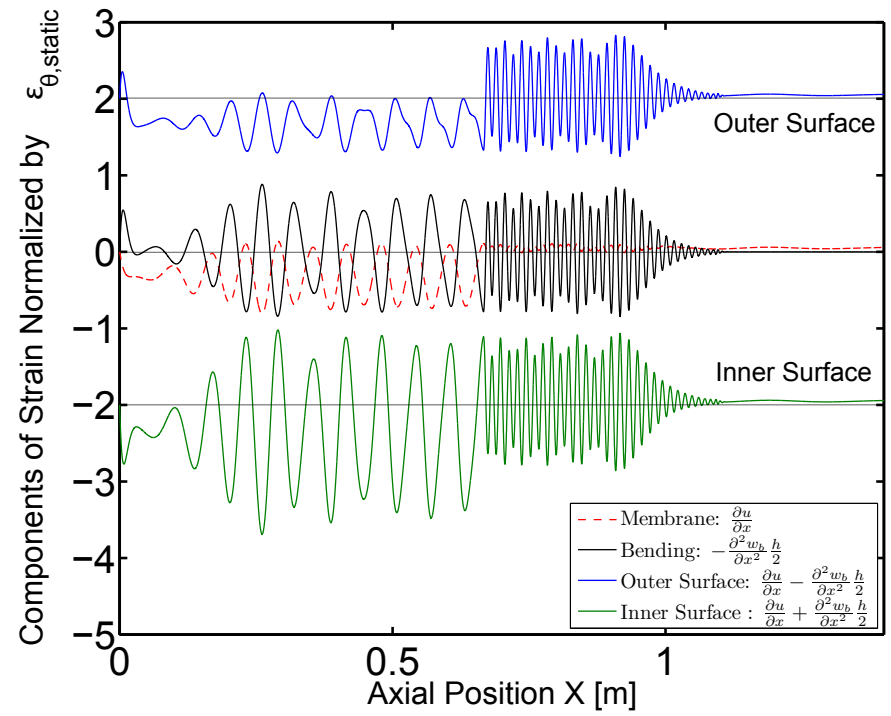

FIGURE 10. Components of axial strain for a tube with $a / h=7$. Bottom and top traces show the total axial strain at the inner and outer surfaces of the tube. Middle two traces show the components of strain due to stretching (dashed) and bending (solid) of the middle surface. For clarity, strains at the inner and outer surfaces have been offset by -2 and +2 , respectively.

plays no role. This plot reveals axial strains at the inner surface of the tube that are more than twice as large as those observed at the middle and outer surfaces.

The reason for the larger strains at the inner surface of the tube can be explained by decomposing the axial strain into the membrane and bending components. These two terms are plotted in Fig. 10 along with the total axial strains at the inner and outer surfaces of the tube. As this figure shows, the components of axial strain due to bending and stretching are of similar amplitude, but are very nearly out of phase. Thus at the outer surface of the tube, there is significant cancellation and the net strain is rather small. At the inner surface of the tube, however, the superposition of these two components results in very large strains.

\section{DISCUSSION OF EXPERIMENTS}

As described in the introduction, the analysis performed in this paper was intended to clarify the behavior of axial strains for thick-walled tubes subjected to detonation loads. In this section, examples of experimental strain measurements are provided and evaluated on the basis of the models developed in this report.

Figure 11 shows axial and hoop strain traces measured by [22] for a thin-walled tube with $a / h=39$. The tube was loaded by a detonation with a Chapman Jouguet (CJ) speed of $U_{C J}=2343 \mathrm{~m} / \mathrm{s}$ and pressure of $P_{C J}=1.5 \mathrm{MPa}$. These measurements are compared with the predictions of the transient shell 


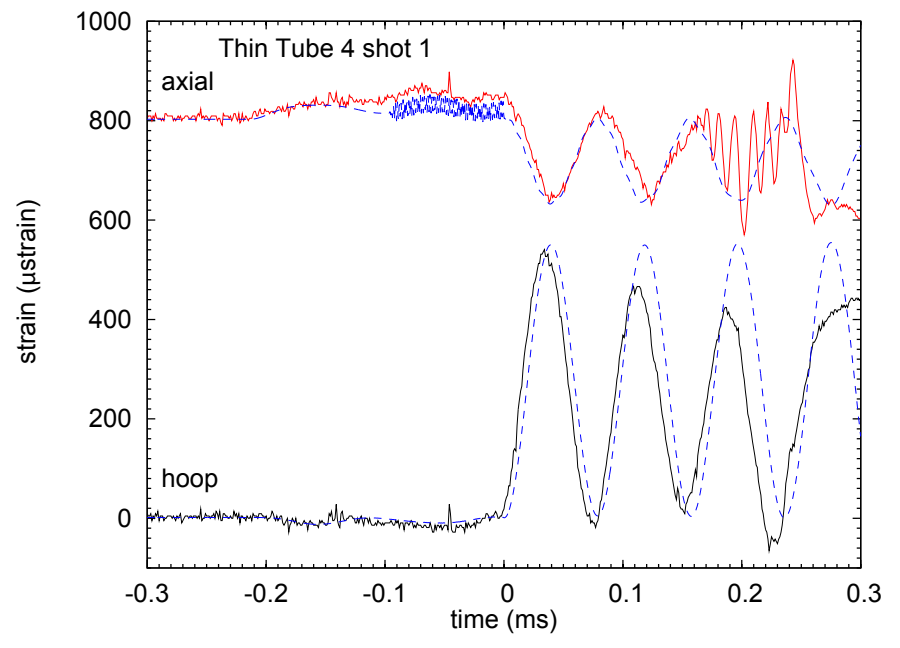

FIGURE 11. Hoop and axial strain measurements for a thin tube $(a / h=39)$ loaded by an internal detonation [22]. Tube is made from C1010 steel with $R_{i}=63.5 \mathrm{~mm}$ and $h=1.65 \mathrm{~mm}$, and is loaded by a detonation with CJ speed and pressure of $U_{C J}=2343 \mathrm{~m} / \mathrm{s}$ and $P_{C J}=1.5 \mathrm{MPa}$. The first critical velocity is $v_{c o}=650 \mathrm{~m} / \mathrm{s}$. Solid lines are measurements, dashed lines are predictions from transient shell model.

model described in Section 5.1 (dashed lines). Very good agreement is observed, especially in the first few cycles following the arrival of the pressure wave at $t=0$. The high-frequency shear waves ahead of the pressure wave are not observed in the experiments due to limitations on spatial and temporal resolution, but the frequency and amplitude of the vibrations behind the wavefront are well predicted. For such a thin tube, the contribution of bending to the axial strains is very small: the ratio of axial to hoop strain is very close to $v=0.3$ and the measured axial strain is out of phase with the hoop strain.

Figure 12 plots axial and hoop strains measured by [6] for a 2 inch, schedule 40 , stainless steel pipe with $a / h=7.2$. The tube was loaded using a detonation in $\mathrm{H}_{2}-\mathrm{N}_{2} \mathrm{O}$ with $\mathrm{CJ}$ speed and pressure of $U_{C J}=2090 \mathrm{~m} / \mathrm{s}$ and $P_{C J}=2.5 \mathrm{MPa}$. The measurements are again compared with the predictions of shell theory, shown as dashed lines. The amplitude and frequency of the hoop strains are close to the expectations from shell theory, but both the qualitative features and quantitative values of the axial strains are significantly different. The wavelength of these vibrations is about $70 \mathrm{~mm}$, or $\lambda / h=18$. For these conditions, the ratio of $\lambda$ to the critical wavelength given by Eq. (18) is about 0.82 , so the membrane and bending components of strain at the outer surface of the tube are expected to nearly cancel out. This behavior is observed in the shell model, but not in the experiments. Instead, the measured axial strains exhibit large vibrations with amplitudes that are nearly the same size as the hoop strains.

This paper has demonstrated that the predictions of shell

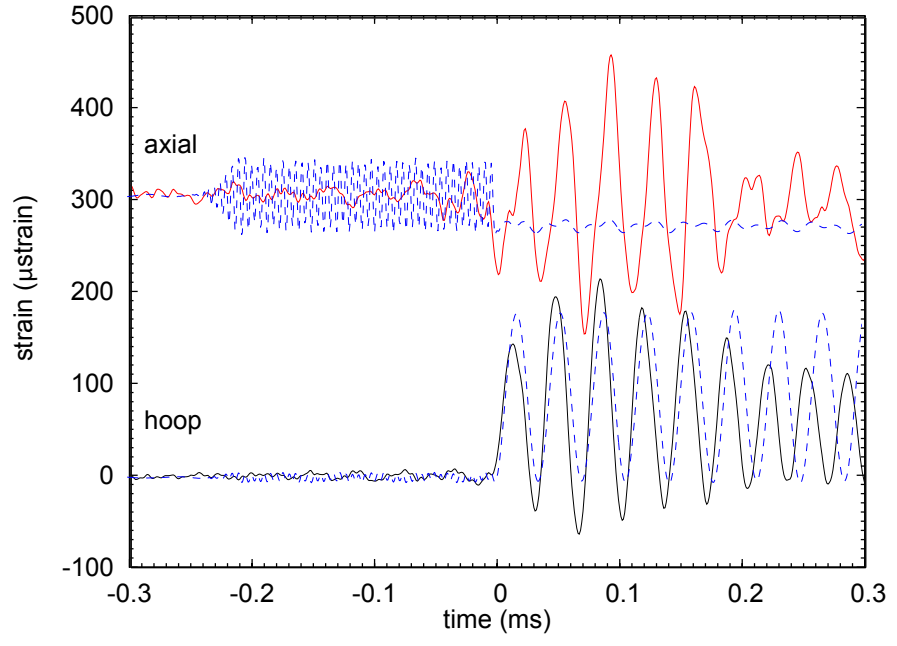

FIGURE 12. Hoop and axial strain measurements for a thick tube $(a / h=7.2)$ loaded by an internal detonation with CJ speed of $U_{c j}=2090 \mathrm{~m} / \mathrm{s}$ and pressure $P_{c j}=2.5 \mathrm{MPa}$. Tube is 2-in schedule 40, 304 stainless steel pipe. Data was recorded in [6], series ES1, test 3, gauges 4 and 5 . The first critical velocity is $v_{c o}=1350 \mathrm{~m} / \mathrm{s}$. Solid lines are measurements, dashed lines are predictions from transient shell model.

models agree quite adequately with the results of highly resolved finite element simulations, even for tubes with wall thickness as large as $a / h=4$. As a result, the discrepancy between the predictions of shell models and the experimental measurements cannot be attributed to the breakdown of the shell model for thick tubes. The large axial strains observed in the experiments also cannot be explained by the through-wall bending effect since the wavelengths of the vibration are too large to produce bending strains as large as those measured in experiment.

The source of the unusual features of the measured strains must therefore be non-ideal conditions that are present in experiments and are not included in the modeling. Several potential causes are the following:

1. Transition to detonation (DDT) after ignition, which occurs inside of the specimen pipe, may produce a non-ideal, transient wave system.

2. Non-axisymmetric features of the pipe specimen, such as weldments and inserts where pressure transducers are mounted, might excite non-axisymmetric modes of vibration that involve coupling between radial and axial motion.

3. Interactions between the vibrating pipe and the mounting hardware used to support the pipe might generate additional wave systems.

4. The cellular structure of the detonation wave may produce to local pressure transients or a non-normal wavefront, which would introduce temporal or spatial variations in pressure near the wavefront. 
Because there are many potential sources of non-ideal behavior, additional experiments conducted under more precisely controlled conditions are needed in order to understand the source of the large axial strains that have been observed.

\section{CONCLUSIONS}

By applying a prescribed middle-surface displacement to both finite element models and shell models, it has been shown that the axial strain at the inner or outer surface of a tube varies inversely with the square of the deformation's wavelength. This scaling is valid until the wavelength decreases below about 10 times the tube's wall thickness, at which point nonlinear behavior is observed and the strains predicted by shell equations become substantially greater than those obtained from finite element models.

Comparisons between dynamic shell models and finite element simulations for shock wave type loads have shown that shell models which include the effects of rotary inertia and transverse shear deformation can adequately predict the response of tubes for most practical tube sizes. For the thickest tubes simulated $(a / h=4)$, vibration frequencies were found to be better predicted than the peak strains, and the peak strains predicted by shell models were up to $20-30 \%$ greater than those obtained using finite element models. Nevertheless, the predictions of shell models adequately capture most features of the response, so such models can be useful tools for understanding the response even of very thick-walled tubes.

The analysis in this paper has demonstrated that the large axial strains which have been observed in experiments cannot be explained by the ideal situation of a shock wave traveling along the inside of a perfect pipe. The magnitude of the through-wall bending strains is not large enough to account for the measured strains, and the breakdown of shell models for thick-walled tubes is also not sufficiently large (nor of the correct sign) to explain those results. Instead, non-ideal features of the experiment such as pipe imperfections or mounting hardware seem to be responsible for these large strains.

\section{ACKNOWLEDGMENT}

This work was motivated by findings of the research program carried out for the Hydrogen in Pipes and Ancillary Vessels (HPAV) study for the Hanford Waste Treatment Plant. Greg Jones of the US Department of Energy, Office of River Protection, Hanford WA, was the technical program manager for the portion of the work done at Caltech. The senior author (JES) benefited from discussions on detonation loading of piping with the members of the HPAV team over the period 2005-2012 while he was associated with the HPAV study. In particular, he would like to acknowledge the very substantial contributions to his understanding of the through-wall bending issue by John Minichiello of Bechtel National Inc, as well as Tom Ligon and David Gross of Dominion Engineering. The present work was funded entirely by the California Institute of Technology; we thank the Office of the Provost and the benefactors of the C.L. "Kelly" Johnson Professorship.

\section{REFERENCES}

[1] Leissa, A. W., 1973. Vibration of shells. Tech. Rep. NASA SP-288, National Aeronautics and Space Administration.

[2] Love, A., 1888. "The small free vibrations and deformation of a thin elastic shell". Philosophical Transactions of the Royal Society A, 179, pp. 491-546.

[3] Beltman, W., Burscu, E., Shepherd, J., and Zuhal, L., 1999. "The structural response of cylindrical shells to internal shock loading". Journal of Pressure Vessel Technology, 121(3), pp. 315-322.

[4] Beltman, W., and Shepherd, J., 2002. "Linear elastic response of tubes to internal detonation loading". Journal of Sound and Vibration, 252(4), pp. 617-655.

[5] Simkins, T., 1994. "Amplification of flexural waves in gun tubes". Journal of Sound and Vibration, 172(2), pp. 145154.

[6] Shepherd, J. E., and Akbar, R., 2009. Piping system response to detonations. results of ES1, TS1 and SS1 testing. Tech. Rep. FM2009-001, Graduate Aeronautical Laboratories California Institute of Technology, April. Revised June 2010.

[7] Gazis, D. C., 1959. "Three-dimensional investigation of the propagation of waves in hollow circular cylinders. i. analytical foundation". Journal of the Acoustical Society of America, 31(5), pp. 568-573.

[8] Gazis, D. C., 1959. "Three-dimensional investigation of the propagation of waves in hollow circular cylinders. ii. numerical results". Journal of the Acoustical Society of America, 31(5), pp. 573-578.

[9] Greenspon, J. E., 1960. "Vibrations of a thick-walled cylindrical shell - comparison of the exact theory with approximate theories". Journal of the Acoustical Society of America, 32(5), pp. 571-578.

[10] Herrmann, G., and Mirsky, I., 1956. “Three-dimensional and shell-theory analysis of axially symmetric motions of cylinders". Journal of Applied Mechanics, 23(4), pp. 563568.

[11] Tang, S., 1965. "Dynamic response of a tube under moving pressure". In Proceedings of the American Society of Civil Engineers. Engineering Mechanics Division. Volume 5, pp. 97-122.

[12] Schiffner, K., and Steele, C. R., 1971. "Cylindrical shell with an axisymmetric moving load". AIAA Journal, 9(1), pp. 37-47. 
[13] Calladine, C. R., 1983. Theory of Shell Structures. Cambridge University Press.

[14] Taylor, R. L. FEAPpv a finite element analysis program: Personal version. Version 3.1, updated March 2012, downloaded from http://www.ce.berkeley.edu/projects/feap/feappv/ on 31 Jan 2013.

[15] Zienkiewicz, O. C., and Taylor, R. L., 2005. Finite Element Method for Solid and Structural Mechanics, 6th ed. Butterworth Heinemann.

[16] Naghdi, P., and Cooper, R., 1956. "Propagation of elastic waves in cylindrical shells, including effects of transverse shear and rotary inertia". Journal of the Acoustical Society of America, 28(1), pp. 56-63.

[17] Lin, C., and Morgan, C., 1956. "A study of axisymmetric vibrations of cylindrical shells as affected by rotatory inertia and transverse shear". Journal of Applied Mechanics, 23(2).

[18] LSTC. LS-DYNA User's Manual, version 971 R6.1.0. Livermore Software Technology Corporation, Livermore, CA. Updated August 2012.

[19] Shepherd, J. E., 2009. "Structural response of piping to internal gas detonation". Journal of Pressure Vessel Technology, 131(3).

[20] Simkins, T., Pflegl, G., and Stilson, E., 1993. "Dynamic strains in a $60 \mathrm{~mm}$ gun tube: An experimental study". Journal of Sound and Vibration, 168(3), pp. 549-557.

[21] Blevins, R. D., 1979. Formulas for Natural Frequency and Mode Shape. Van Nostrand Reinhold Co.

[22] Karnesky, J. A., 2010. "Detonation induced strain in tubes". $\mathrm{PhD}$ thesis, California Institute of Technology, Pasadena, California, May. 